\title{
Envelhecimento: interrelação do idoso com a família e a sociedade
}

\author{
Aging: interrelation of the elderly with the family and society \\ Envejecimiento: interrelación del anciano con la familia y la sociedade
}

Recebido: 27/12/2020 | Revisado: 28/12/2020 | Aceito: 01/01/2021 | Publicado: 02/01/2021

Maria de Fátima Santana de Souza Guerra ORCID: https://orcid.org/0000-0002-2760-8230

Centro Universitário UniAges, Brasil

E-mail: marinaide10@gmail.com

Murilo de Jesus Porto

ORCID: https://orcid.org/0000-0003-2339-8173

Universidade do Estado da Bahia, Brasil

E-mail: murilo.porto@ hotmail.com

Ana Mara Borges Araujo

ORCID: https://orcid.org/0000-0003-4065-241X Centro Universitário UniAges, Brasil E-mail: anamaraborges_@hotmail.com

Jaciara Pinheiro de Souza

ORCID:http://orcid.org/0000-0002-4056-974X Centro Universitário UniAges, Brasil

E-mail: jacipinheirosouza@hotmail.com

Gilvania Piedade Santos

ORCID: https://orcid.org/0000-0003-2435-0198 Centro Universitário UniAges, Brasil E-mail: gilvania.piedade@gmail.com

Welde Natan Borges de Santana

ORCID: https://orcid.org/0000-0001-9781-2934 Centro Universitário UniAges, Brasil E-mail:welder_natan@hotmail.com

Walber Barbosa de Andrade

ORCID: https://orcid.org/0000-0003-0290-5009 Centro Universitário UniAges, Brasil E-mail: blekwalber@hotmail.com

Antero Fontes de Santana

ORCID: https://orcid.org/0000-0002-8944-3216 Centro Universitário UniAges, Brasil E-mail: anterosantana@ hotmail.com

Sandra Regina Santana Silva

ORCID: https://orcid.org/0000-0002-8218-3407 Centro Universitário UniAges, Brasil

E-mail: sandrareginaenfa@hotmail.com

Maciel Borges Nascimento

ORCID: https://orcid.org/0000-0002-9190-6072 Centro Universitário UniAges, Brasil E-mail: macielborges17@gmail.com

\begin{abstract}
Resumo
O processo do envelhecimento ocorre de maneira natural, não depende da vontade do indivíduo, apresentando constantes processos de modificações, onde ocorrem transformações múltiplas, e as mesmas são irreversíveis apesar do avanço da medicina. É heterogêneo e individual, pois cada pessoa tem sua própria velocidade para envelhecer, é deletério, danoso, pois leva a uma perda progressiva das funções. O presente estudo tem como objetivo geral discorrer sobre a relação do idoso com a família e a sociedade, abordando de quem é a responsabilidade do cuidado e da preservação dos direitos do idoso. Trata-se de uma revisão narrativa, com abordagem exploratória qualitativa a nível bibliográfico, por meio da busca eletrônica no Scielo; Google Acadêmico; Lilacs; livros; sites a partir de palavras chaves: Envelhecimento, relação do idoso com a família e a sociedade. Como resultados obtidos, várias publicações mostram que a família, na maioria das vezes, desenvolve sentimentos negativos quanto ao cuidado com o idoso, vendo-o como um empecilho que atrapalha o desenvolver "normal" das atividades diárias. Em contrapartida, para a maioria dos idosos, a família tem um papel essencial na manutenção da sua sobrevivência. Esses fatos permeiam a sociedade, a qual se encontra despreparada para desenvolver uma assistência resolutiva nesse âmbito. A partir dessa revisão bibliográfica, observa-se então a necessidade de serem estabelecidos esquemas assistenciais mais efetivos e
\end{abstract}


dinâmicos, capazes de assistir as demandas crescentes dos idosos e de suas famílias, que em muitos casos passam por problemas nem sempre de conhecimento das equipes de saúde.

Palavras-chave: Envelhecimento; Idoso; Sociedade.

\begin{abstract}
The aging process occurs naturally, it does not depend on the individual's will, presenting constant processes of modifications, where multiple transformations occur, and they are irreversible despite the advancement of medicine. It is heterogeneous and individual, as each person has their own speed to age, it is harmful, harmful, as it leads to a progressive loss of functions. The present study aims to discuss the relationship of the elderly with the family and society, addressing who is responsible for the care and preservation of the rights of the elderly. It is a narrative review, with a qualitative exploratory approach at the bibliographic level, through electronic search in Scielo; Academic Google; Lilacs; books; sites based on keywords: Aging, the elderly 's relationship with family and society. As results obtained, several publications show that the family, in most cases, develops negative feelings regarding the care for the elderly, seeing it as an obstacle that hinders the "normal" development of daily activities. On the other hand, for most elderly people, the family has an essential role in maintaining their survival. These facts permeate society, which is unprepared to develop resolutive assistance in this area. From this bibliographic review, there is then a need to establish more effective and dynamic assistance schemes, capable of assisting the growing demands of the elderly and their families, who in many cases experience problems not always known by the health teams.
\end{abstract}

Keywords: Aging; Old Man; Society.

\title{
Resumen
}

El proceso de envejecimiento ocurre de forma natural, no depende de la voluntad del individuo, presentando constantes procesos de modificaciones, donde ocurren múltiples transformaciones, y son irreversibles a pesar del avance de la medicina. Es heterogéneo e individual, ya que cada persona tiene su propia velocidad de envejecimiento, es dañino, nocivo, ya que conlleva una pérdida progresiva de funciones. El presente estudio tiene como objetivo discutir la relación de las personas mayores con la familia y la sociedad, abordando quién es el responsable del cuidado y preservación de los derechos de las personas mayores. Es una revisión narrativa, con un enfoque exploratorio cualitativo a nivel bibliográfico, mediante búsqueda electrónica en Scielo; Google académico; Lilas; libros; sitios basados en palabras clave: Envejecimiento, relación del anciano con la familia y la sociedad. Como resultados obtenidos, varias publicaciones muestran que la familia, en la mayoría de los casos, desarrolla sentimientos negativos con respecto al cuidado del anciano, viéndolo como un obstáculo que dificulta el desarrollo "normal" de las actividades diarias. Por otro lado, para la mayoría de las personas mayores, la familia tiene un papel fundamental para mantener su supervivencia. Estos hechos impregnan a la sociedad, que no está preparada para desarrollar ayudas resolutivas en este ámbito. A partir de esta revisión bibliográfica, surge entonces la necesidad de establecer esquemas asistenciales más efectivos y dinámicos, capaces de atender las crecientes demandas de las personas mayores y sus familias, quienes en muchos casos experimentan problemas no siempre conocidos por los equipos de salud.

Palabras clave: Envejecimiento; Anciano; Sociedad.

\section{Introdução}

A população idosa no Brasil e no mundo vem crescendo significativamente nos últimos anos. As estatísticas já apontam a evidente mudança no perfil etário na população, que tem como característica principal o aumento da população idosa associada à redução da taxa de natalidade. O envelhecimento da população é inevitável e aponta para a necessidade da sociedade ocupar-se com o processo de envelhecimento ao mesmo tempo em que precisa criar propostas para melhorar as condições de saúde, dignidade e autonomia aos seus idosos (Veras, 2009).

O processo do envelhecimento sofre constantes mudanças, passando por diversas fases, trazendo determinadas mudanças em suas respostas, principalmente, a nível fisiológico (Freitas et al., 2006). Segundo Netto (2007), o envelhecimento pode ser temporariamente uma fase da vida que começa em algum período indeterminado, que segue a maturidade e que se caracteriza pelo declínio progressivo da capacidade funcional e pelo aumento da suscetibilidade a doenças, o que termina por levar a morte.

Diante da crescente demanda de uma população que envelhece e em acordo com os direitos previstos na Constituição de 1988, foi promulgada em 1994, através da Lei 8.842/94, a Política Nacional do Idoso, regulamentada pelo Decreto 1.948/96. Esta política assegurou direitos sociais à pessoa idosa, criando condições que pudessem promover sua autonomia, integração e participação efetiva na sociedade e reafirmando o direito à saúde nos diversos níveis de atendimentos do Sistema 
Único de Saúde - SUS por meio de ações fundamentadas no paradigma da promoção da saúde e da independência da pessoa idosa, direcionando medidas coletivas e individuais de saúde (Brasil, 2010).

A caracterização do processo do envelhecimento é aquele que o conceitua como um processo dinâmico e progressivo, no qual ocorrem alterações morfológicas, funcionais, bioquímicas e psicológicas. Tais mudanças determinam perda progressiva da capacidade de adaptação do indivíduo ao meio ambiente, ocasionando maior vulnerabilidade e maior incidência de processos patológicos que terminam por conduzir à morte (Netto, 2007).

É perceptível a função das políticas públicas de saúde em fazer com que cada vez mais sua população venha atingir uma faixa etária de longevidade em boas condições de saúde. Nesse contexto para considerar saúde de forma holística, torna-se necessária alguma mudança no contexto atual em direção à produção de um ambiente social e cultural mais favorável para população idosa (Brasil, 2007).

Vale ressaltar que conseguir viver por mais tempo nem sempre é sinônimo de viver bem. Desta maneira o presente trabalho fez um levantamento bibliográfico, objetivando discorrer sobre a relação do idoso com a família e a sociedade, abordando de quem é a responsabilidade do cuidado e da preservação dos direitos do idoso.

\section{Metodologia}

O presente trabalho utilizou como metodologia a revisão narrativa, com abordagem exploratória qualitativa. Desta maneira, foram analisados os presentes dados da literatura teórica que deram subsídio para agrupar um vasto leque de propósitos: conceito sobre a temática discutida, avaliação de problemas metodológicos e análise de referenciais teóricos.

A pesquisa qualitativa busca averiguar e abranger o conhecimento de maneira ampla, e por meio de dados estatísticos de determinados fenômenos, analisa a exposição das características de determinada população, caracterizando-a o mais próximo possível da realidade (GIL, 2010).

A pesquisa exploratória cria maior familiaridade com o problema, objetivando a torná-lo mais explícito ou a facilitar a construção de hipóteses. Esse tipo de pesquisa é extremamente flexível, de modo que quaisquer aspectos relativos ao fato estudado têm relevância (Gil, 2010).

A pesquisa de caráter bibliográfica se desenvolve tentando explicar um problema e utiliza certos conhecimentos disponíveis. Ela mostra ao investigador o conhecimento disponível na área, identificando as teorias produzidas, analisando-as e avaliando sua contribuição para auxiliar a compreender ou explicar o problema (Koche, 1999).

Para realização desse trabalho, foram utilizadas as seguintes etapas: verificação da questão temática, estabelecimento dos critérios para a seleção da amostra, análise e interpretação dos resultados e apresentação da revisão. O processo metodológico abordado para a coleta foi a realização de um estudo, através de um levantamento bibliográfico por meio da busca eletrônica no Scielo, Lilacs e Google Acadêmico, a partir de palavras - chaves: Envelhecimento, relação do idoso com a família e a sociedade.

O levantamento de dados utilizou como critérios de inclusão os textos que abordavam o público de estudo (idosos) e a importância da família e da sociedade na assistência aos idosos, não havendo restrição de ano de publicação. Assim foram excluídos trabalhos que fossem publicados em outro idioma que não o português; abordassem outro tema; relatos de casos e entrevistas de jornais. 


\section{Resultados e Discussão}

\section{$O$ processo do envelhecimento: aceitação $x$ desafio}

Há múltiplos fatores associados ao processo de envelhecimento: fatores moleculares, celulares, sistêmicos, comportamentais, cognitivos e sociais. Estes interagem e regulam tanto o funcionamento típico quanto o atípico do indivíduo que envelhece, já que o envelhecimento é um processo que faz parte do nosso desenvolvimento normal, que envolve diversas alterações biológicas, influenciadas por diversos fatores ambientais e socioeconômicos (Palácios, 2004).

O envelhecimento não é um processo unitário, não acontece de modo simultâneo em todo o organismo nem está associado à existência de uma doença. O processo de envelhecimento envolve muitos fatores que devem ser considerados de forma integrada (Palácios, 2004). Muitos avanços têm sido feitos no sentido de apoiar medidas que propiciem um funcionamento saudável nesta faixa etária, bem como de intervir nos fenômenos associados ao envelhecimento patológico, podendo-se citar com exemplo a reabilitação neuropsicológica associada ao tratamento farmacológico (Prado, 2005).

Para a Organização Mundial de Saúde (OMS) o envelhecimento pode ser definido como:

Um processo sequencial, individual, cumulativo, irreversível, universal, não patológico de deterioração de um organismo maduro, próprio a todos os membros de uma espécie, de maneira que o tempo o torne menos capaz de fazer frente ao estresse do meio ambiente (OMS, 2002, p.5).

Neste aspecto podemos discutir o envelhecimento como aceitação ou desafio a partir das condições individuais de cada idoso e de suas necessidades. O envelhecimento pode ser conceituado como um conjunto de modificações morfológicas, fisiológicas, bioquímicas e psicológicas, que determinam a perda progressiva da capacidade de adaptação do indivíduo ao meio ambiente, sendo considerado um processo dinâmico e progressivo. $\mathrm{O}$ declínio das funções orgânicas, manifestadas durante o envelhecimento, tende a aumentar com o tempo, com um ritmo que varia não só de um órgão para outro, mas também entre idosos da mesma idade. Essas diferenças no processo de envelhecimento devem-se às condições desiguais de vida e de trabalho (Papaléo, 2002).

O desafio começa do conjunto de alterações fisiológicas e patológicas vivenciadas pelos idosos, resultando em uma crescente dependência que se traduz por uma necessidade de ajuda indispensável para a realização das atividades elementares da vida. No gral, os idosos não aceitam essas modificações, as quais implicam de forma negativa nesta fase da vida. Vale ressaltar que a dependência não é um estado permanente, mas sim um processo dinâmico, cuja evolução pode se modificar e até ser prevenida ou reduzida (Caldas, 2003). Isso justifica a implementação de programas e práticas que promovam o envelhecimento saudável, em termos de aumento de participação e de redução de custos com cuidados a partir de projetos e praticas intersetoriais.

\section{A Relação do Idoso com a Família e Sociedade}

No cenário nacional, segundo estimativas do IBGE, o número de pessoas com idade igual e/ou superior a 60 anos, é crescente e o país está sendo considerado, um país estruturalmente envelhecido (Ibge, 2009). A explicação desta alteração na forma da pirâmide etária, conforme análise do discurso de Veras (2003), se faz a partir da evolução do controle da natalidade através dos programas de métodos anticonceptivos e dos avanços na área da saúde e biotecnologia que proporcionam o aumento da expectativa de vida da população. Diante desta conjuntura, vemos a necessidade de apreender a atual situação do idoso em seu espaço dentro da sociedade (Oms, 2004; Veras, 2003).

O idoso na sociedade capitalista, na qual a pessoa vale o que produz, é considerado um ser que não gera mais riqueza. Dessa maneira: 
“[...]somos levados a pensar sobre questões relativas à construção da identidade do idoso e de como esta é sentida e vivida pelos indivíduos classificados como velhos" (Mercadante, 2003, p.56).

A sociedade utiliza este modelo social de ser que não produz, atribuindo qualidades negativas aos velhos, negando-os, com isso, um futuro de qualidade. Este modelo também é utilizado pelos próprios idosos para classificar outros, fugindo da própria realidade. $\mathrm{O}$ indivíduo nega a qualidade de velho no momento que a sociedade atribui somente aspectos negativos desta condição. Com o decorrer dos anos o conceito de idoso para a sociedade foi sendo alterado (Debert, 1999).

[...] antes do século XVIII, a velhice era considerada ridícula; no século XIX, sábia; no século XX, a velhice como conceito biológico e moral desaparece e a pressão social exerce-se no sentido de negar a velhice enquanto tal valorizando-se a pessoa que consegue disfarçá-la fisicamente (velhos bem conservados) e / ou psicologicamente (velhos de espírito jovem) [...] (Barreto, 1992, p. 23).

De maneira geral, a sociedade, não valoriza a velhice, conservando apenas o conceito de que os velhos devem ser respeitados, porém este respeito vem travestido de características típicas do indivíduo jovem, tanto física, como moral e psicologicamente. Os idosos, dentro da atual circunstância da sociedade, têm ambientes próprios propostos ao seu atendimento, entretenimento e manutenção da "identidade". Esses lugares são os centros de convivência para idosos, grupos de terceira idade e ainda programas institucionais de atendimento ao idoso (Debert, 1999).

Por muito tempo, em nossa sociedade, grande parte dos indivíduos que envelheciam vivia um processo de isolamento, alguns pela fragilização das relações sociais quando deixavam o mundo do trabalho e outros, em geral mulheres, pela diminuição das obrigações familiares à medida que os filhos conquistavam sua independência (Brasil, 2010).

Socialização é um termo amplo que indica que o ser humano, desde que nasce, não apenas está sujeito às influências da sociedade de que participa e ajuda a construir, como também a influencia. É universal nas sociedades com elevados níveis de mudança social, que obrigam seus membros de qualquer idade a uma constante assimilação de conhecimentos e de formas de atuar (Moragas, 2010, p. 101).

Desde as décadas de 1960 e 1970, cada vez mais pessoas estão vivendo o processo de envelhecimento sem sucumbir à imposição de afastamento, porque têm sido seres ativos capazes de dar respostas originais aos desafios que encontram em seu cotidiano, redefinindo sua experiência para, assim, se contrapor aos estereótipos ligados à velhice (Brasil, 2010).

Assim, é necessário encarar os novos desafios propostos, mas entendemos que com eles devem coexistir atividades voltadas para a luta pela cidadania e garantia dos direitos, de maneira complementar e integrada às de lazer e socioculturais, que são formas lúdicas de Associação, fundamentais à natureza humana e bases da sociabilidade. O resultado do trabalho coletivo desenvolvido com os idosos é de importância inquestionável e constitui relevante alternativa para a ressocialização, melhoria da qualidade de vida e redefinição de seu lugar na sociedade (Brasil, 2010).

Nas décadas de 1980 e 1990, as universidades brasileiras começaram a abrir espaço para programas de educação destinados a adultos maduros e a idosos, por meio das UNATIS, cujo trabalho está intrinsecamente ligado ao desenvolvimento da sociabilidade e à educação permanente. Esse tipo de educação se justifica por sua natureza potencializadora, por seu caráter de compensar lacunas do ensino formal, por favorecer o conhecimento a respeito do processo de envelhecimento, o engajamento social, o sentido de auto eficácia, o bem-estar subjetivo, a aproximação de gerações, a capacitação para exigir direitos e a autonomia de pensamento. Além disso, a educação é apontada como determinante de uma velhice bem-sucedida (Brasil, 2010).

A educação para adultos maduros e idosos deve pretender no mínimo incrementar os saberes e os conhecimentos práticos, o saber fazer, o aprender e seguir aprendendo, e possibilitar o crescimento contínuo, as relações sociais e a participação social (Cachioni, 2008, p. 46). 
A partir da década de 1980, o SESC, atento às mudanças da sociedade e em face das exigências desse "novo idoso", incentivou a criação das Escolas Abertas da Terceira Idade. Estes núcleos de idosos se organizam em torno de atividades permanentes que incentivam o convívio e o desenvolvimento de habilidades nas relações interpessoais (Brasil, 2010).

Os programas das UNATIS inserem-se no conceito de educação permanente proposto pela UNESCO, como aquela em que o aprendizado deve fazer-se ao longo de todo o curso da vida, de modo constante, interativo e cumulativo, para acompanhar as mudanças rápidas e contínuas da sociedade moderna. As diferentes formas de educação têm de abranger os processos que levam aos indivíduos de todas as idades um conhecimento dinâmico do mundo, dos outros e de si mesmos.

As instituições, tanto públicas quanto privadas, costumam oferecer os cursos como programas de extensão universitária, sem exigência de pré-requisitos de escolaridade, além da alfabetização, para pessoas acima de 45 anos. Estruturam-se em regime sequencial ou modular, em geral com disciplinas e atividades de conteúdo psicológico, sociológico e médico sobre o envelhecimento, atualização cultural, noções básicas sobre informática e atividades de lazer, físicas e de expressão artística.

O interesse pela questão dos aposentados passou a ganhar maior destaque em 1990, com a Constituição de 1988 e as transformações ocorridas na Previdência Social e nas políticas de aposentadoria, que tenderam à universalização de direitos. Um dos marcos dessa visibilidade foi a criação das associações de aposentados. Organizadas nacionalmente, elas assumem o papel de interlocutoras políticas dos aposentados e pensionistas na reivindicação de garantia de direitos e manutenção do poder aquisitivo envolvem-se na luta contra a imagem negativa desse segmento e estabelecem alianças com outros setores desfavorecidos da sociedade em defesa da distribuição de renda e dos direitos sociais perante o Estado.

Seu trabalho consiste na prestação de informações atualizadas sobre os direitos dos aposentados e na condução de processos contra a Previdência Social. Elas também publicam informativas e mobilizam a participação dos associados em manifestações por seus direitos, além de esporadicamente promover congressos, conferências e encontros. Denunciando as injustiças sociais, o movimento trouxe à tona o modo de vida carente de muitos idosos e suas constantes agruras em filas diante de agências do INSS, nos corredores dos hospitais públicos e nas instituições de longa permanência (Debert, 1999).

A experiência de militantes nessas associações faz com que os indivíduos sejam representados coletivamente como aposentados ativos, ex- trabalhadores. Substitui a imagem de encargos para a família pela de provedores, engajados na luta que beneficiará todas as gerações e não apenas voltados à sobrevivência e satisfação pessoal. Ser militante da causa do aposentado não é ser idoso. Idoso é o outro por quem eles lutam para ajudar (Simões, 2007).

Atualmente, as associações perderam espaço na cena política, porém continuam sendo formas de socialização, pois ser militante traz senso de pertencimento e afirmação. Constituem espaços coletivos, organizados de modo solidário, nos quais novas estratégias políticas permitem atuar em defesa de interesses e valores comuns quanto à questão dos direitos dos aposentados, pensionistas, idosos e, em consequência, de toda a sociedade (Debert, 1999).

A dinâmica da participação nas associações estabelece laços sociais que envolvem convivência menos intensa entre os participantes, mas suas manifestações e reivindicações políticas beneficiam, em princípio, todos, qualquer que seja sua renda (Debert, 1999).

Essas formas sistemáticas de sociabilidade, inovadoras e marcadas, sobretudo pelo encontro interacional no espaço público, são tendências crescentes e têm importância inquestionável. Elas, entretanto, não envolvem todos os idosos, mas sim importante parcela daqueles que usufruem o direito à aposentadoria, majoritariamente mulheres, que se beneficiaram da emancipação feminina, da autonomia e da igualdade de direitos entre os sexos, com maior poder de deliberação sobre a própria 
vida. São homens e mulheres saudáveis, dispostos a iniciar atividades novas, estabelecer contatos e reunir-se fora da família. Fazem parte de uma geração em sintonia com o tempo presente, reinventando os modos de envelhecer (Motta, 2007).

Tais experiências se traduzem, ainda, em oportunidade de trabalho para profissionais e técnicos envolvidos com a questão do idoso, que devem incentivar a manutenção dessas conquistas. Cabe-lhes também a tarefa de refletir com os centros, grupos, associações e universidades sobre a necessidade de uma integração social mais ampla para o alcance da cidadania plena, colaborando para que os passos seguintes sejam dados, ou seja, alcançar o convívio e a solidariedade entre as gerações nos espaços públicos e privado e o estreitamento de forças pelo entrosamento mais efetivo entre diferentes grupos de idosos, favorecendo seu movimento social e político e a redefinição de seu lugar na sociedade.

A família tem um posicionamento peculiar com relação à pessoa idosa. Logo que o idoso é considerado, em muitas famílias, como um peso a ser carregado, que não tem mais utilidade. É um empecilho que atrapalha o desenvolver "normal" das atividades diárias de uma família, como passeios, programas de finais de semana, etc. Em contrapartida, para a maioria dos idosos, a família tem um papel relativo na sua sobrevivência, sendo que este papel relativo refere-se à liberdade de decisões que o senil deseja tomar, mas a família teme que ele não tenha autonomia suficiente para decidir o que é melhor para si (Mercadante, 2003).

Existe ainda um pensamento de senso comum que o envelhecimento é sinônimo de dependência econômica, entretanto os dados do IBGE indicavam que 62,4\% dos idosos eram os responsáveis pelos domicílios brasileiros, onde a principal fonte de renda dos idosos é a aposentadoria, para ambos os sexos (Ibge, 2000).

A família geralmente satisfaz as inúmeras necessidades de seus integrantes, sejam elas físicas psíquicas e sociais, porém, nas últimas décadas essa satisfação das necessidades tem se modificado profundamente com a transformação de uma sociedade agrária em uma sociedade urbana industrializada; e, muitas dessas necessidades estão sendo atendidas por organizações alheias à família, embora as decisões fundamentais ainda continuem sendo tomadas em seu interior, quando a família não pode por algum motivo tomar essas decisões fundamentais o Estado decide sobre a educação e saúde dos membros (Moragas, 2010).

A rotina das relações familiares mantém oculta a complexidade e sutileza de seu emaranhado, muito superior ao das organizações formais mais elaboradas. A maior parte dos idosos casados se apoia na própria família de procriação, da qual eles são a primeira geração em ordem cronológica (Moragas, 2010, p. 156).

As necessidades fisiológicas de alimentação na velhice são muito importantes, refeições em família garantem a qualidade e o equilíbrio de uma dieta balanceada, o que não ocorre quando a idosa mora sozinha. A saúde tem sido com frequência o ponto mais frágil do idoso, e a família constitui um intermediário muito útil na busca de recursos de saúde para eles. Também é comum que os mecanismos de defesa e de equilíbrio da personalidade sejam ameaçados por diversas tensões, e a família pode proporcionar um meio mais favorável para manter a identidade pessoal do idoso e o manter fora dos riscos do meio ambiente (Moragas, 2010).

Dentre as diversas funções e papéis que podem ser desenvolvidos pelos diversos membros familiares, duas estão sempre presentes: o prover e o cuidar. Esse funcionamento nem sempre é harmônico, e isso pode contribuir para que possam surgir doenças nos membros da família, em especial nos idosos, que acabam perdendo aos poucos as funções previamente definidas (Brasil, 2007). 


\section{Considerações Finais}

A partir desse levantamento bibliográfico, observa-se então a necessidade de serem estabelecidos esquemas assistenciais mais efetivos, resolutivos e dinâmicos, capazes de assistir as demandas crescentes dos idosos e de suas famílias, que em muitos casos passam por problemas nem sempre de conhecimento das equipes de saúde.

Várias publicações mostram que a família, na maioria das vezes, desenvolve sentimentos negativos quanto ao cuidado com o idoso, vendo-o como um empecilho que atrapalha o desenvolver "normal" das atividades diárias. Em contrapartida, para a maioria dos idosos, a família tem um papel essencial na manutenção da sua sobrevivência. Esses fatos permeiam a sociedade, a qual se encontra despreparada para desenvolver uma assistência resolutiva nesse âmbito.

Destarte, deve-se planejar e se atentar para esse fato com foco na busca por maior qualidade de vida, independência, promoção da saúde mental, física, social para o idoso. Percebe-se que o meio jurídico é o alicerce para a proteção do idoso, entretanto, é de suma importância a conscientização, a nível populacional e com ênfase na família, acerca da necessidade de se proporcionar meios que promova qualidade de vida.

Pertinente ao futuro, essa revisão bibliográfica contribuirá na sensibilização acerca do processo de envelhecimento e da necessidade de reflexão e disponibilização de soluções e situações que possibilite qualidade de vida aos idosos. Ainda esse trabalho se constitui um arcabouço, fonte de conhecimento para proporção de soluções as particularidades do processo de senescência.

\section{Referências}

Brasil. (2007). Uma proposta de política pública de atividade física para idosos. Texto Contexto Enfermagem 16(3) 387-98.

Brasil. (2010). Envelhecimento e saúde da pessoa idosa. Série A. Normas e Manuais Técnicos Cadernos de atenção básica, 19. Brasília - DF.

Brasil. (1989). Constituição Federal de 1988, Brasília: Senado Federal, Secretaria Especial de Editoração e Publicações: Câmara dos Deputados, Coordenação de Publicações.

Brasil. (1994). Lei n. 8.842, de 4 de janeiro de 1994. Dispõe sobre a Política Nacional do Idoso. Brasília.

Brasil. (2003). Ministério da Saúde. Estatuto do Idoso. Brasília: Ministério da Saúde.

Brasil. (2006). Ministério da Saúde. Revista Brasileira Saúde da Família Ministério da Saúde Secretaria de Atenção à Saúde Departamento de Atenção Básica. Brasília.

Brasil. (2013). Ministério da Saúde. Conselho Nacional de Saúde. Resolução no 466, de 12 de dezembro de 2012 . Publicada no DOU nº 12 - quinta-feira, Seção $1-$ P. 59

Brasil. (2011). Ministério da Saúde. Abc do SUS doutrinas e princípios. Brasília/DF: http://biblioteca.planejamento.gov.br/biblioteca-tematica-1/textos/saudeepidemias-xcampanha.".

Barreto, M. L. F. (1992). Admirável Mundo Velho. Ártica.

Bueno. F. M. G. (2006). O enfermeiro e a construção da autonomia profissional no processo de cuidar. Revista brasileira de enfermagem.59(2). Brasília Mar./Apr.

Caldas, C. P. (2003). Envelhecimento com dependência: responsabilidades e demandas da família. Caderno Saúde Pública. 19 (3): $773-81$.

Koche, J. C. (1999). Fundamentos de metodologia científica. UCS.

Cachioni, M. (2008). Gerontologia educacional/ educação gerontológica. In: Neri, A.L. (Org.). Palavras-chave em gerontologia, 92-94. (3a ed.) Alínea.

Debert, G. G. (1999). Velhice e sociedade, Papirus Editora, Campinas.

Freitas, G. F., Oguisso, T. (2010). Ética no Contexto da Prática de Enfermagem. Med. book Editora Científica. Rio de janeiro-RJ.

Gil, A. C. (2010). Como elabora projetos de pesquisa (5a ed.), Atlas.

Ibge. (2015). Censo 2000. Recuperado de: <www.tabnet.datasus.gov.br>.

Ibge. (2008). Síntese de indicadores sociais: uma análise das condições de vida da população brasileira. Rio de Janeiro: IBGE. http://www.ibge.gov.br/ home/estatistica/populacao/condicaodevida/indicadoresminimos/sintese >. 
Research, Society and Development, v. 10, n. 1, e3410111534, 2021

(CC BY 4.0) | ISSN 2525-3409 | DOI: http://dx.doi.org/10.33448/rsd-v10i1.11534

Ibge. (2009). Indicadores sociodemográficos $e$ de saúde no Baneiro.

http://www.ibge.gov.br/home/estatistica/populacao/condicaodevida/indicadoresminimos/sintese>.

Mercadante, E. F. (2003) Velhice: a identidade estigmatizada. Revista Serviço Social e Sociedade; (75), 55-72. São Paulo: Cortez.

Moragas. R. M. (2010). Gerontologia social: envelhecimento e qualidade de vida. (3a ed.), São Paulo: Paulinas.

Motta, L. B., Aguiar, A. C. (2007). Novas competências profissionais em saúde e o envelhecimento populacional brasileiro: integralidade, interdisciplinaridade e intersetorialidade. Ciência e Saúde Coletiva.

Oms. Organização Mundial Da Saúde. (2002). Versão Em Português Dos Instrumentos De Avaliação De Qualidade De Vida (Whoqol).

Palácios, J. (2004). Mudança e Desenvolvimento Durante a Idade Adulta e a Velhice. Em C. Coll, J. Palacios, \& A. Marchesi. Desenvolvimento Psicológico e Educação Psicologia Evolutiva (2a.ed.), Artmed.

Papaléo, N. M. (2007). Gerontologia. Atheneu.

Prado, S. D., Sayde, J. D. (2005). A Pesquisa sobre Envelhecimento Humano no Brasil: Grupos e Linhas de Pesquisa. <http://Www.Scielo.Br/Pdf/Csc/V9n1/19823.Pdf>.

Simões, A. L. A. et al. (2007). Humanização na saúde: enfoque na atenção primária. Texto \& Contexto Enfermagem, Florianópolis,16(3), 439-444.

Veras, R. P. (2003). A longevidade da população: desafios e conquistas. Revista Serviço Social e Sociedade; n.75. São Paulo: Cortez.

Veras, R. (2009). Envelhecimento populacional contemporâneo: demandas, desafios e inovações. Rev. Saúde Pública, São Paulo, 43(3). 